\title{
Decision analysis modelling of costs and outcomes following cefepime monotherapy in Canada
}

\author{
Michael T Halpern mD PhD, Ruth E Brown mS MHSA, Martine Drolet B PHarm MBa, \\ SONJA V SORENSEN MSPH, LIONEL A MANDELL MD FRCPC
}

\begin{abstract}
MT HALPERN, RE BROWN, M DROLET, SV SORENSEN, LA MANDELL. Decision analysis modelling of costs and outcomes following cefepime monotherapy in Canada. Can J Infect Dis 1997;8(1):19-27.

OBJECTIVE: To evaluate the comparative cost of treatment and intermediate outcomes (percentage resistant organisms, days in hospital, etc) among cefepime and alternative parenteral antibiotics used for empiric monotherapy. DESIGN: Decision analysis model, based on published literature, clinical trial results and information from infectious disease clinicians.

SETTING: A Canadian tertiary care hospital.

INTERVENTION: Comparison of cefepime, ceftazidime, ceftriaxone, cefotaxime and ciprofloxacin in the treatment of lower respiratory tract infections, urinary tract infections, skin/soft tissue infections, septicemia and febrile neutropenia. MAIN RESULTS: Cefepime treatment results in the lowest average cost per patient when used as initial empiric therapy for lower respiratory tract infections and for skin/soft tissue infections. Cefepime therapy is among the lowest cost treatments for the other infectious disease conditions and has the lowest cost for a weighted 'average' condition. Sensitivity analysis indicates that model results are most sensitive to duration of hospitalization.

CONCLUSIONS: Initial empiric monotherapy with cefepime for serious infectious disease conditions may result in cost savings compared with alternative parenteral agents.
\end{abstract}

Key Words: Antibiotics, Cefepime, Costs, Decision analysis, Economic models

\section{Modèle d'analyse décisionnelle des coûts et des résultats d'une monothérapie par céfépime au Canada}

OBJECTIFS : Évaluer le coût comparatif du traitement et des résultats à moyen terme (pourcentage d'organismes résistants, jours d'hospitalisation, etc.) entre le céfépime et d'autres antibiotiques administrés par voie parentérale en monothérapie empirique.

MODÈLE : Modèle d'analyse décisionnelle basé sur la littérature publiée, les résultats d'essais cliniques et des renseignements fournis par des infectiologues.

CONTEXTE : Un hôpital canadien de soins tertiaires.

INTERVENTION : Comparaison du céfépime, de la ceftazidime, du ceftriaxone, du céfotaxime et de la ciprofloxacine dans le traitement des infections des voies respiratoires basses, des infections urinaires, des infections des tissus mous et de la peau, de la septicémie et de la neutropénie fébrile.

PRINCIPAUX RÉSULTATS : Le traitement par céfépime donne lieu au coût moyen le plus bas par patient lorsqu'il est utilisé en traitement empirique initial dans les cas d'infection des voies respiratoires basses et d'infection de la peau et des tissus mous. Le traitement par céfépime se trouve parmi les traitements les moins coûteux dans le cas des autres 
types d'infection et représente le traitement le moins coûteux pour ce qui est de la moyenne pondérée. L'analyse de sensibilité indique que les résultats du modèle sont les plus sensibles pour ce qui est de la durée d'hospitalisation.

CONCLUSIONS : La monothérapie empirique initiale au moyen de céfépime dans les cas de graves infections peut entraîner une économie si on la compare avec d'autres agents administrés par voie parentérale.

$\mathrm{T}$ here has been a dramatic increase in the prevalence of serious infections caused by antibiotic-resistant bacteria (1). As a consequence, many patients may receive empiric antibiotic therapy with agents to which the infecting organisms are resistant. This may lead to alteration of the initial empiric therapy, prolonged hospitalization and increased costs. Further, many of the broad-spectrum antibiotics that do not show high levels of resistance are associated with frequent or serious side effects.

Cefepime, a fourth generation parenteral cephalosporin with broad antibacterial activity, is classified as an extended broad-spectrum cephalosporin (2). Cefepime can be used for a variety of infections, including community-acquired and nosocomial pneumonia, bacteremia/septicemia, complicated and uncomplicated urinary tract infections, complicated skin and skin structure infections, intra-abdominal infections and certain gynecological infections (2). The use of cefepime in nosocomial infections may be especially advantageous because of the low incidence of cefepime-resistant organisms $(3,4)$. Compared with many older cephalosporins, cefepime has increased activity against Gram-positive organisms, higher affinity for multiple penicillin-binding proteins and greater resistance to beta-lactamases (2). Few side effects beyond gastrointestinal disturbances have been reported $(3,5)$.

Limited health care resources and concern over containing hospital costs have caused decision makers to call increasingly for cost effectiveness studies of new medications. A clinical decision analysis model to examine the costs and outcomes associated with cefepime therapy compared with therapy with alternative parenteral antibiotics in Canada was developed. The design of and results from this model are described below.

\section{METHODS}

The cefepime decision analysis model compares the costs and outcomes of cefepime with those of ceftazidime, ceftriaxone, cefotaxime and ciprofloxacin (intravenous) in treating five types of nosocomial or complicated infections: lower respiratory tract infections (LRTI), predominantly pneumonia; septicemia; urinary tract infections (UTI), predominantly pyelonephritis; skin/soft tissue infections; and febrile neutropenia. It models the most efficient manner to treat patients (ie, which starting regimen results in the shortest length of stay, fewest adverse events and lowest cost) based on specified initial parameters for clinical outcomes and medical resource utilization.

A basic assumption of this model is that the initial choice of antibiotic (among the five compared here) will not significantly influence mortality. However, therapy with certain antibiotics may initially lead to greater clinical efficiency, such as more rapid patient improvement or less need to alter medica- tions, than therapy with other antibiotics. Therefore, while overall mortality is assumed to be identical among the different initial antibiotics, intermediate model outcomes, such as the percentage of patients with known resistance, the percentage showing sufficient clinical improvement and the percentage experiencing adverse drug events, will be different for each starting medication. These differences result in variations in required length of hospitalization, treatment regimens and overall costs.

In the model (Figure 1), patients start therapy with one of the above five antibiotics as initial empiric treatment. On day 2, patients' bacterial culture and sensitivity results as well as their clinical status are evaluated. Patients are then placed into one of two categories: those showing sufficient clinical improvement who remain on their initial monotherapy; or those requiring alternative or additional antibiotics due to resistant organisms or lack of clinical improvement. Patients requiring additional or alternative therapy remain hospitalized for an extended period (Table 1). At day 4, the model evaluates patients requiring treatment for or a switch in medication due to adverse drug events (ADEs). Patients switching to alternative therapy on day 4 because of ADEs require hospitalization for an additional period (Table 1).

Baseline length of hospitalization for all conditions except febrile neutropenia was obtained from the 1993-94 Canadian Institute for Health Information database (Hay Healthcare Consulting Group, Toronto). These values represent averages for Metro Toronto hospitals. The value for length of hospital stay for febrile neutropenia was taken from Tomiak et al (6). Length of hospitalization for each clinical conditions associated with different model outcomes is presented in Table 1.

Information for the model parameter values and treatment patterns was obtained from results of clinical trials carried out by Bristol-Myers Squibb, the available medical literature and communications with Canadian infectious disease practitioners. To the greatest extent possible, the model was based on information from documented sources (published or unpublished literature); expert opinion was used only when needed information could not be otherwise obtained. Model parameters obtained from the published sources or databases included cost and dosing of antibiotics; other health care costs (eg, cost per hospital day); incidence of bacterial resistance; probability of obtaining definitive bacterial cultures; probability of clinical improvement; incidence and type of adverse drug events; and baseline length of hospitalization.

The initial model parameters were the probability of definitive bacterial resistance and the probability (among patients with sensitive or unknown organisms) of clinical improvement (Figure 1). Definitive bacterial resistance was defined as the probability of the infecting organism being resistant to the selected antibiotic multiplied by the probability that culture 


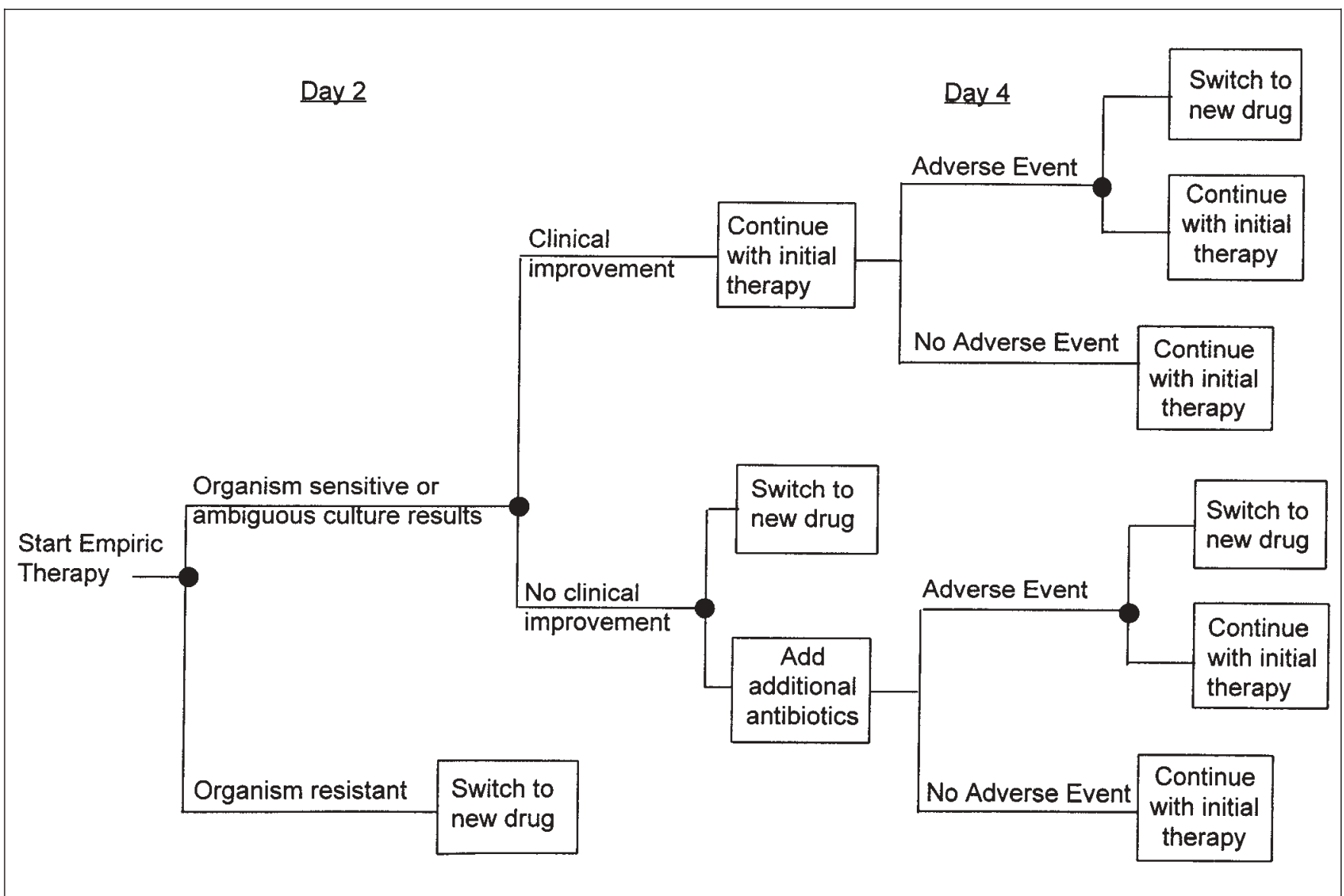

Figure 1) Cefepime decision analysis model. Patients begin empiric monotherapy on day 0. On day 2, bacterial culture and sensitivity results are evaluated; patients with resistant organisms are switched to new antibiotic drugs, while those with sensitive organisms or ambiguous culture results have their clinical status evaluated. Patients with sufficient clinical improvement continue on their initial therapy; patients whose improvement is not adequate are switched to new medication or receive further antibiotics in addition to their current therapy. On day 4, patients who remained on their initial therapy (either alone or in combination with additional medications) may experience adverse events. Patients experiencing adverse events may switch to an alternative medication or continue with their current therapy

results identify the infectious organism. The likelihood of obtaining definitive culture results (cultures that isolated the causative organism) varied for each of the five infectious disease conditions. The probability of obtaining definitive culture results was assumed to be $100 \%$ for UTI and septicemia because these conditions are defined by the presence of identified bacteria. For the other infectious diseases, the probability of obtaining definitive culture and sensitivity results was based on the meta-analysis of the studies used to estimate the likelihood of clinical improvement. From this analysis, it was estimated that definitive culture results were obtained in $52.4 \%$ of LRTI, $70.6 \%$ of skin and soft tissue infections and $40.2 \%$ of febrile neutropenia.

The probability of having a resistant organism was determined from the frequency distribution of bacteria causing each condition and the resistance of the causative organisms to each antibiotic. For all infectious conditions except febrile neutropenia, bacterial frequency distributions were based on values from the National Nosocomial Infection Surveillance (NNIS) system, conducted by the Centers for Disease Control and Prevention, Altanta, Georgia (7). As NNIS system data do not include febrile neutropenia, bacterial frequency distributions for this condition were derived from Carlisle et al (8).

The likelihood of a bacterial species showing resistance to one of the selected antibiotics was derived from Canadian data whenever possible. A majority of values were derived from Scriver et al (9). Bacterial resistance for Gram-negative organisms not included in this study was derived from Chamberland et al (10). Canadian resistance figures for Gram-positive and anaerobic organisms for the antibiotics under study were not available; therefore, American data from Jones et al (11), Murray et al (12), Neu (13), and Washington et al (14) were used. It should be noted that various studies were used to estimate bacterial resistance because no single published report compares the resistance of all the organisms with all of the antibiotics under study.

Bacterial resistance for any selected infectious disease condition and antibiotic combination was determined by the product of the frequency of bacteria causing the condition and the resistance of each causative bacteria to the antibiotic. For example, Staphylococcus aureus were responsible for $17.3 \%$ of LRTI, and $21.0 \%$ of $S$ aureus showed resistance to cefepime. 
TABLE 1

Length of hospital stay (days) for model outcomes by infectious disease condition

\begin{tabular}{|c|c|c|c|c|c|}
\hline Model condition(s) & Type of therapy & $\begin{array}{l}\text { LRTI, skin/soft } \\
\text { tissue infection }\end{array}$ & UTI & Septicemia & $\begin{array}{c}\text { Febrile } \\
\text { neutropenia }\end{array}$ \\
\hline $\begin{array}{l}\text { Base case (organisms sensitive, clinical } \\
\text { improvement, no ADEs) }\end{array}$ & Initial therapy & 4.7 & 4.4 & 5.2 & 10 \\
\hline \multirow{3}{*}{$\begin{array}{l}\text { Organism sensitive, clinical } \\
\text { improvement, ADE leading to } \\
\text { alternative therapy }\end{array}$} & Initial therapy & 4 & 4 & 4 & 4 \\
\hline & New therapy & 2 & 2 & 2 & 7 \\
\hline & Total & 6 & 6 & 6 & 11 \\
\hline \multirow{3}{*}{$\begin{array}{l}\text { Organism resistant or no clinical } \\
\text { improvement, switch to alternative or } \\
\text { additional therapy }\end{array}$} & Initial therapy & 2 & 2 & 2 & 2 \\
\hline & $\begin{array}{l}\text { Added/alternative } \\
\text { therapy }\end{array}$ & 4.7 & 4.4 & 5.2 & 10 \\
\hline & Total & 6.7 & 6.4 & 7.2 & 12 \\
\hline
\end{tabular}

ADE Adverse drug event; LRTI Lower respiratory tract infections; UTI Urinary tract infections

TABLE 2

Probability of bacterial resistance*

\begin{tabular}{lccccc}
\hline Antibiotic & UTI & LRTI & Skin and soft tissue & Bacteremia & Febrile neutropenia \\
\hline Cefepime & 0.232 & 0.133 & 0.246 & 0.199 & 0.182 \\
Ceftazidime & 0.254 & 0.171 & 0.317 & 0.323 & 0.270 \\
Ceftriaxone & 0.265 & 0.180 & 0.300 & 0.322 & 0.216 \\
Cefotaxime & 0.241 & 0.182 & 0.234 & 0.192 & 0.175 \\
Ciprofloxacin & 0.036 & 0.069 & 0.129 & 0.158 & 0.127 \\
\hline
\end{tabular}

*In vitro probability of bacterial resistance based primarily of Scriver et al (9). LRTI Lower respiratory tract infections; UTI Urinary tract infections

TABLE 3

Probability of clinical effectiveness of antibiotics on susceptible infections*

\begin{tabular}{lccccc}
\hline Antibiotic & UTI & LRTI & Skin and soft tissue & Bacteremia & Febrile neutropenia \\
\hline Cefepime & 0.890 & 0.780 & 0.840 & 0.710 & 0.747 \\
Ceftazidime & 0.830 & 0.840 & 0.800 & 0.620 & 0.566 \\
Ceftriaxone & 0.763 & 0.651 & 0.706 & 0.769 & 0.695 \\
Cefotaxime & 0.900 & 0.633 & 0.649 & 0.768 & 0.773 \\
Ciprofloxacin & 0.883 & 0.748 & 0.818 & 0.862 & 0.275 \\
\hline
\end{tabular}

*Probability of clinical effectiveness based on Cefepime New Drug Application database (US Food and Drug Administration submission 50-679) or meta-analysis results. LRTI Lower respiratory tract infections; UTI Urinary tract infections

Therefore, $S$ aureus contributed 0.173 times 0.210 or $3.6 \%$ resistance to cefepime in LRTI. These product terms were summed over all causative organisms to produce the net estimated resistance for each infectious disease and antibiotic combination. Bacterial resistance values are presented in $\mathrm{Ta}$ ble 2 .

If bacterial cultures indicated organisms resistant to the initial empirical antibiotic therapy, patients were switched to alternative therapy. If culture results showed sensitive organisms or were ambiguous, the patient's clinical improvement was evaluated. The likelihood of patients showing clinical improvement on cefepime or ceftazidime for each infectious disease except febrile neutropenia was based on data from the Cefepime New Drug Application database (US Food and Drug Administration submission 50-679). The database contained information on 991 cefepime patients and 672 ceftazidime patients.

For the other three antibiotics in each of the infectious disease conditions and for cefepime and ceftazidime used to treat febrile neutropenia, the likelihood of clinical improvement was based on meta-analysis results from published studies. Relevant literature was located through automated bibliographic databases, review of references from identified articles and suggestions by the clinical experts involved in this study. All identified reports were evaluated in terms of quality and appropriateness of research methodology, and only those findings from scientifically credible studies were included. These were published studies which specified study inclusion and exclusion criteria; defined the treatment protocols, types of infections and outcome measures; and accounted for all patients entering the study. Study design was a mixture of randomized trials and nonrandomized case series reports.

In evaluating the likelihood of clinical improvement, the following criteria were used to select published studies for meta-analysis: studies reporting monotherapy for each of the selected infectious disease conditions; all patients with known 
TABLE 4

Total daily antibiotic dose by infectious disease

\begin{tabular}{lccccc}
\hline Antibiotic & $\begin{array}{c}\text { Lower respiratory } \\
\text { tract infections }\end{array}$ & $\begin{array}{c}\text { Urinary tract } \\
\text { infections }\end{array}$ & $\begin{array}{c}\text { Skin/soft tissue } \\
\text { infections }\end{array}$ & Septicemia & Febrile neutropenia \\
\hline Cefepime $(\mathrm{g})$ & 2 & 1.5 & 2 & 4 & 6 \\
Ceftazidime $(\mathrm{g})$ & 3 & 1.5 & 3 & 6 & 6 \\
Cefotaxime $(\mathrm{g})$ & 5 & 2 & 6 & 4 & 8 \\
Ceftriaxone $(\mathrm{g})$ & 2 & 1 & 2 & 4 & 2 \\
Ciprofloxacin $(\mathrm{g})$ & 0.6 & 0.4 & 0.4 & 0.4 & 0.6 \\
\hline
\end{tabular}

resistant organisms excluded, or data presented in a manner that allowed exclusion of results from patients with known resistant organisms; no prior antibiotic therapy within $72 \mathrm{~h}$ of the start of monotherapy, with the exception of gasterointestinal decontamination therapy for patients undergoing bone marrow transplantation; and results presented in terms of clinical cures. Note that patients with resistant organisms were switched to alternative medications earlier in the model (as discussed above); thus, in determining the probability of clinical improvement, all patients with known resistant organisms had already been excluded.

A total of 49 publications were used, involving 4396 patients or episodes (febrile neutropenia was generally reported in terms of episodes while other infectious conditions were reported in terms of patients, each having a single episode). Random effects meta-analysis (15) was used to provide a more accurate estimate of the impact of antibiotic therapy on all patients, not just patients included in the studies; this technique produces estimates that can be generalized more readily.

Clinical improvement likelihood values used in the model are presented in Table 3 . These values are not the overall effectiveness of each antibiotic for each condition, but represent the likelihood of patients infected with susceptible organisms experiencing a clinical cure.

Patients remaining on their initial antibiotic (either alone or combined with additional therapy) may experience treatment-related ADEs. The probabilities of ADEs due to cefepime or ceftazidime are based on values from the cefepime NDA database; probabilities of ADEs due to cefotaxime, ceftriaxone or ciprofloxacin are based on weighted averages from the studies included in the clinical effectiveness meta-analysis.

Table 4 provides information on antibiotic dosing. Dosing information was derived from the Cefepime NDA database or from median values of doses used in the articles included in the clinical effectiveness meta-analysis. While these dosages represent the median values used in published studies, they may not reflect the most common current clinical practices (eg, ciprofloxacin 200 to $300 \mathrm{mg}$ intravenously every $12 \mathrm{~h}$ was used in the model for febrile neutropenia and septicemia rather than $400 \mathrm{mg}$ intravenously every $12 \mathrm{~h}$ ).

Costs used in the model are presented in Table 5. Medication costs were based on Canadian hospital acquisition prices. Costs for medical tests (cultures and antibiotic sensitivities) were obtained from the Ontario Medical Association 1992 Schedule of Fees (16), while daily hospital costs were obtained from Statistics Canada (17). Costs for preparation and admini-
TABLE 5

Model costs

\begin{tabular}{lc}
\hline Model parameter & Cost $\mathbf{( \$ ) ^ { * }}$ \\
\hline Antibiotic cost per gram & \\
Cefepime & 14.70 \\
Ceftazidime & 12.83 \\
Ceftriaxone & 33.50 \\
Cefotaxime & 9.20 \\
Ciprofloxacin & 82.50 \\
Bacterial cultures & \\
Blood & 24.77 \\
Sputum & 18.16 \\
Skin & 20.64 \\
Urine & 16.51 \\
Antibiotic sensitivities & 9.91 \\
Intravenous medication prepara- & 7.83 \\
tion and administration (per dose) & \\
One day of hospitalization & 521.84 \\
\hline
\end{tabular}

* 1994 Canadian \$

stration of intravenous medications were obtained from Dranitsaris et al (18). All costs used in the model are in 1994 Canadian dollars.

A number of sensitivity analyses were conducted to evaluate the robustness of the model, its underlying assumptions and estimates of critical model parameters. In these analyses, attention focused on determining the effects of changing the value of estimates where there is considerable uncertainty.

Three university-based Canadian infectious disease consultants provided input regarding alternative and additional treatment regimens following detection of resistance or lack or clinical effectiveness, including length of therapy and treatment patterns for adverse drug events, type of treatment and likelihood of withdrawal from the inducing medication. Choice of alternative antibiotic therapies was based on consensus recommendations by the clinical consultants; while these may not represent the choice of second-line therapy in all cases, they serve as illustrative examples of likely alternative choices. It is not possible to include all possible agents that may be used for second-line therapy. Expert opinion was only used when no generalizable data were available to describe general treatment patterns. Without parameters based on expert opinion, it would not have been possible to model the costs and outcomes associated with parenteral antibiotic therapy. 
TABLE 6

Average modeled cost (in 1994 CDN \$) per lower respiratory tract infection patient*

\begin{tabular}{lccccc}
\hline Cost category & Cefepime & Ceftazidime & Ceftriaxone & Cefotaxime & Ciprofloxacin \\
\hline Hospitalization & 2,700 & $2,651(-49)$ & $2,824(124)$ & $2,872(172)$ & $2,673(-27)$ \\
Medications & 185 & $214(29)$ & $353(168)$ & $279(94)$ & $253(68)$ \\
Adverse events & 116 & $148(32)$ & $174(58)$ & $89(-27)$ & $226(110)$ \\
Intravenous supplies & 81 & $112(31)$ & $57(-24)$ & $117(36)$ & $90(9)$ \\
Bacterial culture and sensitivities & 28 & $28(0)$ & $28(0)$ & $28(0)$ & $28(0)$ \\
Average cost per patient & 3,110 & $3,153(42)$ & $3,437(327)$ & $3,385(274)$ & $3,270(160)$ \\
\hline
\end{tabular}

*Values in parentheses represent marginal costs compared with cefepime

TABLE 7

Average cost (in 1994 CDN \$) per patient for four infectious diseases*

\begin{tabular}{lccccc}
\hline Average total cost per patient & Cefepime & Ceftazidime & Ceftriaxone & Cefotaxime & Ciprofloxacin \\
\hline Skin/soft tissue infections & 3,122 & $3,292(170)$ & $3,459(337)$ & $3,417(295)$ & $3,175(53)$ \\
Urinary tract infections & 2,946 & $3,066(120)$ & $3,147(201)$ & $2,914(-32)$ & $2,876(-70)$ \\
Septicemia & 3,689 & $3,957(269)$ & $4,008(319)$ & $3,595(-94)$ & $3,541(-148)$ \\
Febrile neutropenia & 6,841 & $6,996(156)$ & $6,672(-168)$ & $6,749(-92)$ & $7,052(212)$ \\
\hline
\end{tabular}

*Values in parentheses represent marginal costs compared with cefepime

\section{RESULTS}

Results from the base case model are presented in Tables 6 and 7 . These tables present the average cost per patient treated empirically with a single initial antibiotic for a specified disease condition. The average costs consist of the costs associated with both successes and failures, including costs of bacterial resistance, lack of clinical improvement and ADE. In Table 6, costs from the LRTI model are broken into five categories. Hospitalization costs refer to the cumulative hospital charges. Medication costs include those for antibiotics, including initial therapy, alternative therapy and additional therapy. ADEs costs represent the average cost per patient associated with adverse events, including both treatment and sequelae; in other words, this category contains the average costs associated with hospitalization following change of therapy due to ADEs and excess treatment costs for superinfection and pseudomembranous colitis, when appropriate. Costs for bacterial culture and sensitively were identical among the different therapies within an infectious disease because all patients had a single culture and sensitivity assay at the start of therapy. Numbers in parentheses represent the marginal differences between costs for cefepime and each comparison antibiotic for that cost category. Average costs for the other infectious disease conditions are presented in Table 7 without the breakdown into cost categories.

Comparing the five infectious disease conditions, average costs can be ranked in order of baseline length of hospitalization. Costs for UTI were lowest, followed by LRTI and skin/soft tissue infections, septicemia and febrile neutropenia. The importance of length of hospitalization is further examined in the sensitivity analysis section.

Table 8 presents values for an average infectious disease state, based on weighted costs across the five infectious disease models. Here, costs were weighted by the incidence of each of the five infectious disease conditions based on values from the 1993 Medical Marker Studies Limited audit of Canadian hospitals with 500 or more beds. The relative weights were LRTI $51 \%$; UTI $17 \%$; septicemia $22 \%$; skin/soft tissue infections $16 \%$; and febrile neutropenia $8 \%$. In this average state, the baseline length of hospitalization was 5.8 days. Cefepime had the lowest cost per patient of all antibiotics compared. The average per patient cost of cefepime and ciprofloxacin was similar. The higher cost of ciprofloxacin, resulting from adverse events, was partially balanced by lower hospital costs (due to lower bacterial resistance, but partially offset by lower clinical effectiveness).

Sensitivity analysis: Sensitivity analyses for LRTI and septicemia were performed. Patients with these conditions may undergo prolonged courses of therapy because of resistant organisms, resulting in greater use of medical resources and hence increased costs. While the sensitivity analyses focused on these two conditions, similar marginal changes would be observed using any of the infectious diseases. Sensitivity analyses were performed for a number of model parameters.

Model costs: Sensitivity analysis results from changing model costs for LRTI and septicemia were very similar. Even a large change in the price of the initial antibiotic (doubling or halving the price) had relatively moderate impact on the total per patient costs (less than 12\%), reflecting the relatively small proportion of costs made up by medication. In contrast, increasing or decreasing daily hospital costs by only $20 \%$ had a proportionally large impact on overall patient costs for all five antibiotics examined (16\% to $18 \%$ ). The impact of adding a charge for an infectious disease consultation, as would be the case in American hospitals whenever therapy was changed or ADEs occur, had little impact on the average costs (2\%).

Clinical parameters: Doubling or halving bacterial resistance had only a minor effect on per patient costs, ranging from $0 \%$ to $3 \%$. Increasing or decreasing clinical effectiveness to the boundaries of the $90 \%$ confidence intervals also had a rela- 
TABLE 8

Average modelled cost (in 1994 CDN \$) per weighted infectious disease condition*

\begin{tabular}{lccccc}
\hline Cost category & Cefepime & Ceftazidime & Ceftriaxone & Cefotaxime & Ciprofloxacin \\
\hline Hospitalization & 3,358 & $3,389(31)$ & $3,467(109)$ & $3,477(119)$ & $3,265(-93)$ \\
Medications & 292 & $323(31)$ & $454(162)$ & $325(33)$ & $298(6)$ \\
Adverse events & 154 & $200(46)$ & $224(70)$ & $113(-42)$ & $299(145)$ \\
Intravenous supplies & 107 & $139(33)$ & $77(-29)$ & $151(44)$ & $112(5)$ \\
Bacterial culture and sensitivities & 34 & $34(0)$ & $34(0)$ & $34(0)$ & $44(0)$ \\
Total & 3,945 & $4,086(141)$ & $4,257(312)$ & $4,099(154)$ & $4,008(63)$ \\
\hline *The five infectious disease conditions were weighted by their relative incidences. Values in parentheses represent marginal costs compared with cefepime
\end{tabular}

TABLE 9

Threshold change for alternative antibiotics to result in equivalent cost with cefepime for the average weighted infectious disease condition*

\begin{tabular}{|c|c|c|c|c|}
\hline Threshold condition & Ceftazidime & Ceftriaxone & Cefotaxime & Ciprofloxacin \\
\hline Increase in cost per gram (1994 CDN \$) & 11.44 & 24.11 & 12.19 & 4.54 \\
\hline Increase in bacterial resistance (\%) & 21.5 & 45.4 & 22.9 & 8.5 \\
\hline Decrease in clinical effectiveness (\%) & 15 & 31.8 & 16 & 5.9 \\
\hline Increase in baseline length of stay (days) & 0.26 & 0.55 & 0.28 & 0.10 \\
\hline
\end{tabular}

${ }^{*}$ Cost for the average weighted infectious disease condition is presented in Table 8

tively small impact on per patient costs, approximately $4 \%$. Changing the baseline length of hospitalization by two days had the largest impact on average patient costs, with changes averaging 34\% for LRTI and 31\% for septicemia.

ADEs rates: Sensitivity analysis results from modifying ADEs rates (doubling and halving these rates) show only a mild effect on per patient costs. This effect was fairly uniform across all antibiotics included in this study and was similar for LRTI and septicemia.

Threshold analysis: As an additional sensitivity analysis, a threshold analysis was performed for cefepime compared with the other antibiotics in the mean (weighted) infectious disease state (as presented in Table 8). For this analysis the cost, bacterial resistance, clinical effectiveness or length of stay for cefepime were altered until the average per patient cost was identical to the comparator. Changes in the parameters necessary to reach this threshold are presented in Table 9. The threshold analysis results for ceftazidime and cefotaxime were similar. For either, the cost per gram of cefepime (\$14.70 in the base models) was nearly doubled before average patient costs were the same. In order for the average per patient costs associated with initial cefepime therapy to equal the corresponding cost for initial ceftazidime or cefotaxime therapy, bacterial resistance to cefepime had to increase by over $20 \%$ or the rate of clinical success using cefepime had to decrease by $15 \%$ to $16 \%$. Examining the threshold for baseline length of hospitalization, patients initially receiving cefepime had to be hospitalized for at least an additional 0.26 days before average patient costs were the same.

Results for the threshold analysis of ceftriaxone are approximately double those of ceftazidime or cefotaxime. For cefepime costs to equal those of ceftriaxone, the unit cost of cefepime had to almost triple, the bacterial resistance to ce- fepime had to increase by over $45 \%$, the clinical effectiveness of cefepime had to decrease by over $31 \%$ or baseline length of hospitalization had to increase by over one-half day per patient. Ciprofloxacin showed the least difference from cefepime. The unit cost of cefepime had to increase by about one third, the bacterial resistance had to increase by $8.5 \%$, the clinical effectiveness had to decrease by $5.9 \%$ or baseline length of hospitalization had to increase by 0.1 days for average cefepime costs to equal those of ciprofloxacin.

\section{DISCUSSION}

This model has examined the costs and outcomes of cefepime, ceftazidime, cefotaxime, ceftriaxone and ciprofloxacin use in LRTI, UTI, skin/soft tissue infections, septicemia and febrile neutropenia. While we assumed that the eventual outcomes (cure versus death) would not be affected by the choice of initial empiric monotherapy, intermediate outcomes - likelihood of bacterial resistance, clinical failure, and/or adverse drug events - clearly are affected. Because these three outcomes influence patient treatment pathways and the length of hospitalization, they control overall hospitalization costs.

As seen in Table 6, cefepime has the lowest average cost per patient as an initial therapy for LRTI. Combined with the impact of resistant organisms and adverse events on hospital stay, ceftazidime patients have a slightly lower mean length of hospitalization and, thus, lower hospital costs compared with cefepime patients.

However, this lower cost for ceftazidime was offset by higher costs for medication, ADE and intravenous supplies (cefepime is administered twice daily in LRTI while ceftazidime is administered three times daily).

For skin/soft tissue infections, use of cefepime as the initial empiric therapy again resulted in the lowest average per pa- 
tient cost of any of the antibiotics included in this study. For UTI, cefepime had lower average cost than ceftazidime and ceftriaxone, but higher costs than ciprofloxacin and cefotaxime. The lower average cost of ciprofloxacin was due to the very low level of bacterial resistance to this drug in UTI (approximately one-sixth of cefepime), combined with a clinical effectiveness comparable with cefepime. Although this results in significantly lower hospitalization costs for ciprofloxacin, these decreased costs were partially offset by higher ADE costs. Lower medication costs, reflecting a $60 \%$ relative decrease in daily dose for cefotaxime compared with $25 \%$ for cefepime, and lower adverse events led to a slightly decreased total cost for cefotaxime compared with cefepime.

In the treatment of septicemia, compared with cefepime, ceftazidime and ceftriaxone had significantly higher costs while cefotaxime and ciprofloxacin had somewhat lower costs. The lower average costs for cefotaxime and ciprofloxacin were due to differences in medication costs (reflecting the higher dosing of cefepime for septicemia) and hospitalization costs (reflecting the lower bacterial resistance and higher clinical effectiveness of cefotaxime and ciprofloxacin).

The lower cost of cefotaxime was partially offset by a higher intravenous supply cost (reflecting four times daily dosing of cefotaxime compared with twice daily for cefepime); however, cefotaxime may alternatively be administered three times daily. The lower cost of ciprofloxacin was partially offset by a higher ADE cost.

For febrile neutropenia, initial therapy with cefepime resulted in less cost than starting therapy with ceftazidime or ciprofloxacin but more than with ceftriaxone or cefotaxime. The savings for cefotaxime were driven by lower medication and ADE costs; however, this savings was partially offset by greater hospitalization and intravenous supply costs. The lower cost of ceftriaxone was primarily because of lower costs for medication and intravenous supplies.

A number of assumptions were made in designing this model and performing the analysis. First, we assumed that each of the five medications would be used initially as monotherapy. This may not completely reflect 'real-world' practices, because some of the medications in certain disease conditions may be used initially in combination with other agents, such as aminoglycosides. However, it is reasonable to compare the costs and outcomes of each medication used (initially) alone because monotherapy is often a preferred therapeutic practice to decrease the risk of drug interactions and adverse events.

We also realized that some of the medications studied herein would not be used for certain indications (eg, ceftriaxone as initial therapy for febrile neutropenia). These scenarios were included to allow comprehensive comparisons of the medications under all modelled disease conditions. Further, a number of commonly used medications were not included in this model (eg, trimethoprim-sulfamethoxazole for UTI). Particularly in the cases of UTI and skin/soft tissue infections, the selected antibiotics may limit the usefulness of these results in clinical practice. However, we restricted the model to serious infections that required hospitalization and parenteral antibiotics. For these infections, the use of the broad-spectrum parenteral antibiotics included in the model is more appropriate initial therapy than many commonly used oral agents.

Second, we assumed that patients would receive parenteral antibiotics throughout their hospitalization. It is possible that patients may receive parenteral therapy only until a successful response occurs, and then are switched to oral medications. While many studies have not reported a decrease in length of hospitalization caused by switching to oral therapy, a number of studies have shown such a decrease (19-21). Because the number of days of hospitalization mainly drove the cost for patients in this model, changes in treatment patterns resulting in decreased length of hospital stay have a significant impact on total cost. It is unknown whether a stepdown to oral therapy will affect length of hospitalization in Canada. In any event, switching to oral therapy will have the same impact on length of hospitalization for all patients treated successfully with initial antibiotic therapy. Thus, the magnitude of difference in total cost between cefepime and alternative antibiotics will be generally similar to that presented here and will be even greater for certain model scenarios.

Finally, it is also important to note that we have not examined all possible medications likely to be used in the infectious diseases under study. Numerous other agents or agent combinations could have been included. However, the five agents included in this study are common choices for treating infectious diseases, and it is important to compare their relative costs and outcomes.

Using this model, cefepime has the lowest average cost per patient for two infectious disease states, LRTI and skin/soft tissue infections. Cefepime, cefotaxime and ciprofloxacin have similar average costs for UTI. For septicemia and febrile neutropenia, cefepime costs range from $\$ 92$ to $\$ 168$ more than the least expensive antibiotic. The relative cost efficiency of cefepime can be attributed to cefepime's mix of low levels of bacterial resistance, a high rate of clinical efficacy and a favorable ADE profile, which results in shorter hospital stays and lower hospital costs.

Sensitivity analysis identified daily hospital cost and length of hospitalization as the main factors influencing patient costs in this model. We currently are unable to determine whether the baseline length of hospitalization (days in hospital with an optimal clinical course) is influenced by the choice of initial antibiotic therapy. Future research should examine this question to develop more accurate estimates of the comparative costs of therapy.

A previously published study (22) modelled the cost of therapy with cefepime versus ceftazidime for serious infectious diseases. The present study included a broader data set and additional comparator medications as well as treatment patterns and costs specific to Canada. Most importantly, our study separately examined the components of successful treatment with initial antibiotic therapy, bacterial resistance and clinical effectiveness. Explicitly evaluating the likelihood of observing resistant organisms based on the choice of initial empiric therapy is especially relevant, given the recent increases in multidrug-resistant bacteria. Differences in bacterial resistance are also seen in different geographic regions 
and different types of hospitals. Thus, bacterial resistance information specific to Canada produces results that are more useful for accurately determining the relative benefits of selected parenteral antibiotics. The flexibility of this model will allow users to incorporate resistance data as well as costs and clinical outcome probabilities from their own institutions to

\section{REFERENCES}

1. Goldmann DA, Weinstein RA, Wenzel RP, et al. Strategies to prevent and control the emergence and spread of antimicrobial-resistant mircoorganisms in hospitals. JAMA 1996;275:234-40.

2. Sanders CC. Cefepime: The next generation? Clin Infect Dis 1993; 17:369-79.

3. Barradell LB, Harriet MD. Cefepime: A review of its antibacterial activity, pharmacokinetic properties and therapeutic use. Drugs 1994;47:471-505.

4. Vanhoof R, Nulens E, Nyssen H-J, Hannecart-Pokorni E. Comparative in vitro activity of cefepime and four extended-spectrum beta-lactams on 1,251 aminoglycoside-resistant Gram-negative hospital strains. Chemotherapy 1992;38:225-31.

5. Bristol-Myers Squibb. Maxipime. Canadian product monograph, 1995.

6. Tomiak AT, Yau JC, Huan SD, et al. Duration of intravenous antibiotics for patients with neutropenic fever. Ann Oncol 1994;5:441-5.

7. Emori TG, Gaynes RP. An overview of nosocomial infections, including the role of the microbiology laboratory. Clin Microbiol Rev 1993;6:428-42.

8. Carlisle PS, Gucalp R, Wiernik PH. Nosocomial infections in neutropenic cancer patients. Infect Control Hosp Epidemiol 1993;14:320-4.

9. Scriver SR and the Canadian Antimicrobial Resistance Study Group. Comparative activity of several antimicrobial agents against nosocomial Gram-negative rods isolated across Canada. Can J Infect Dis 1995;6:76-82.

10. Chamberland S, L'Ecuyer J, Lessard C, et al. Antibiotic susceptibility profiles of $941 \mathrm{Gram}$-negative bacteria isolated from sepcicemic patients throughout Canada. Clin Infect Dis 1992;15:615-28.

11. Jones RN, Barrett MS, Erwin ME. In-vitro activity of FK-037, a new parenteral cephalosporin. J Antimicrob Chemother 1994;33:137-44. determine institution-specific total costs and outcomes of specific antibiotic treatment regimens.

ACKNOWLEGEMENTS: This research was supported by Bristol-Myers Squibb Canada.

12. Murray PR, Jones RN, Allen SD, Erwin ME, Fuchs PC, Gerlach EH. Multilaboratory evaluation of the in vitro activity of 13 beta-lactam antibiotics against 1474 clinical isolates of aerobic and anaerobic bacteria. Diagn Microbiol Infect Dis 1993; 16:191-203.

13. Neu HC. Cephalosporins-cefotaxime 10 years later, a major drug with continued use. Infection 1991;19(Suppl):S309-15.

14. Washington JA, Jones RN, Gerlach EH, Murray PR, Allen SD, Knapp CC. Multicenter comparison of in vitro activities of FK-037, cefepime, ceftriaxone, ceftazidime, and cefuroxime. Antimicrob Agents Chemother 1993;37:1696-700.

15. Laird NM, Mosteller F. Some statistical methods for combining experimental results. Int J Tech Assess Health Care 1990;6:5-30.

16. Ontario Medical Association. 1992 Schedule of Fees. Toronto: Ontario Medical Association, 1992.

17. Statistics Canada. Health Reports 1993;5:250. Cat No 82-003.

18. Dranitsaris G, Tran TM, McGeer A, Narine L. Pharmacoeconomic analysis of empirical therapy with ceftazidime alone or combination antibiotics for febrile neutropenia in cancer patients. PharmacoEconomics 1995;7:49-62.

19. Bailey RR, Lynn KL, Robson RA, Peddie BA, Smith A. Comparison of ciprofloxacin with netilmicin for the treatment of acute pyelonephritis. NZ Med J 1992;105:102-3.

20. Ehrenkranz NJ, Nerenberg DE, Shultz JM, Slater KC. Intervention to discontinue parenteral antimicrobial therapy in patients hospitalized with pulmonary infections: Effect on shortening patient stay. Infect Control Hosp Epidemiol 1992;13:21-32.

21. Ramirez JA, Srinath L, Ahkee S, Huang A, Ram MJ. Early switch from intravenous to oral cephalosporins in the treatment of hospitalized patients with community-acquired pneumonia. Arch Intern Med 1995;155:1273-6.

22. Paladino JA. Cost-effectiveness comparison of cefepime and ceftazidime using decision analysis. PharmacoEconomics 1994;5:505-12. 


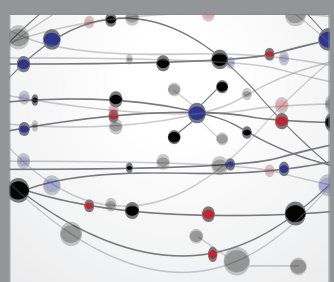

The Scientific World Journal
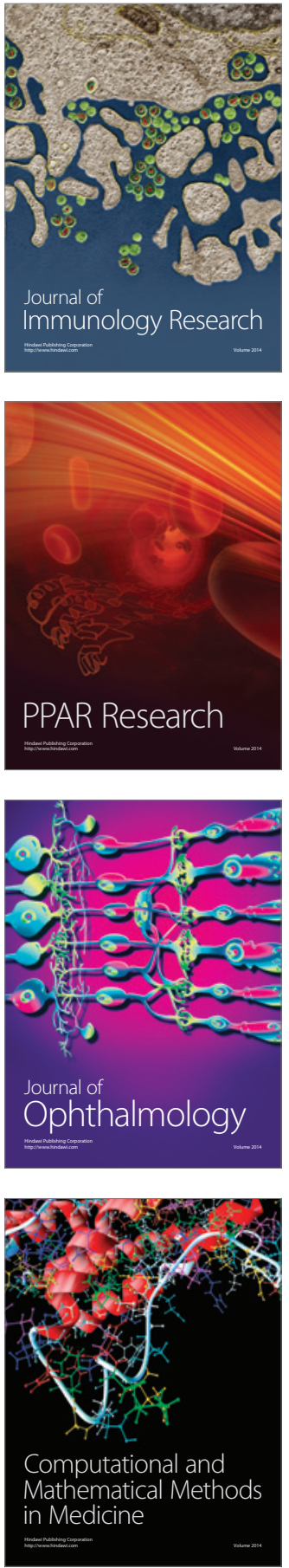

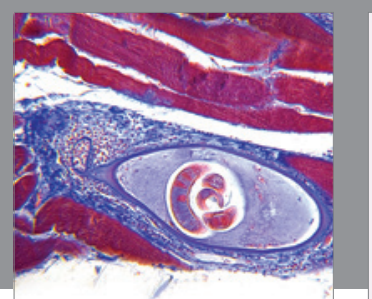

Gastroenterology Research and Practice

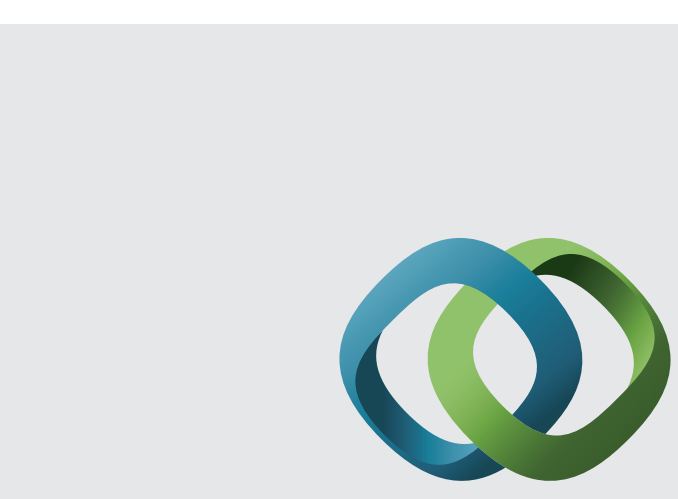

\section{Hindawi}

Submit your manuscripts at

http://www.hindawi.com
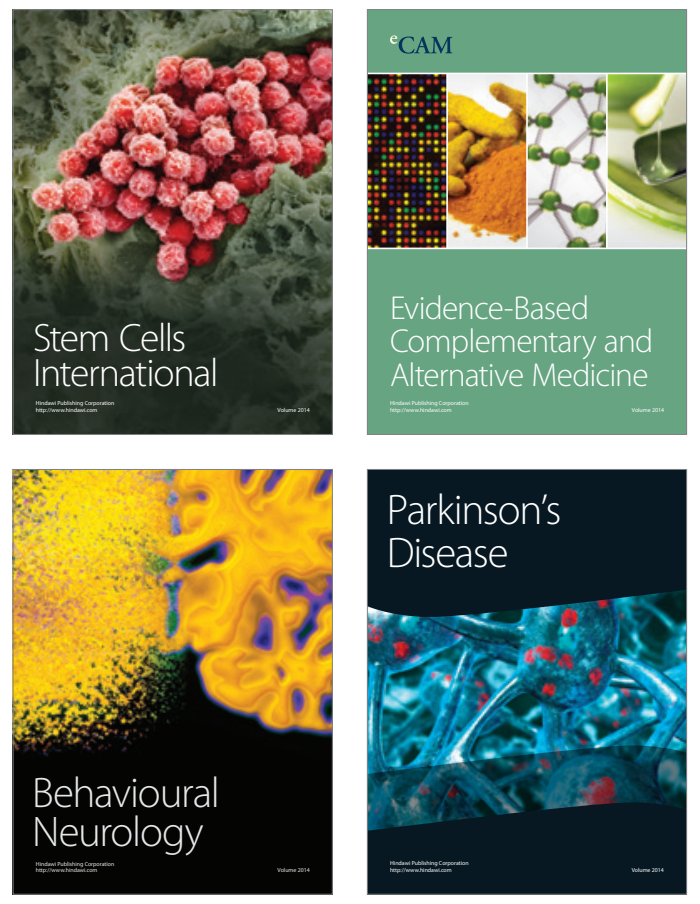
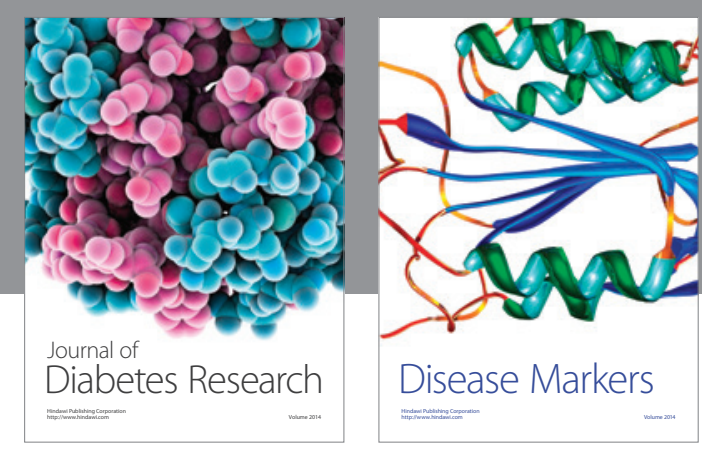

Disease Markers
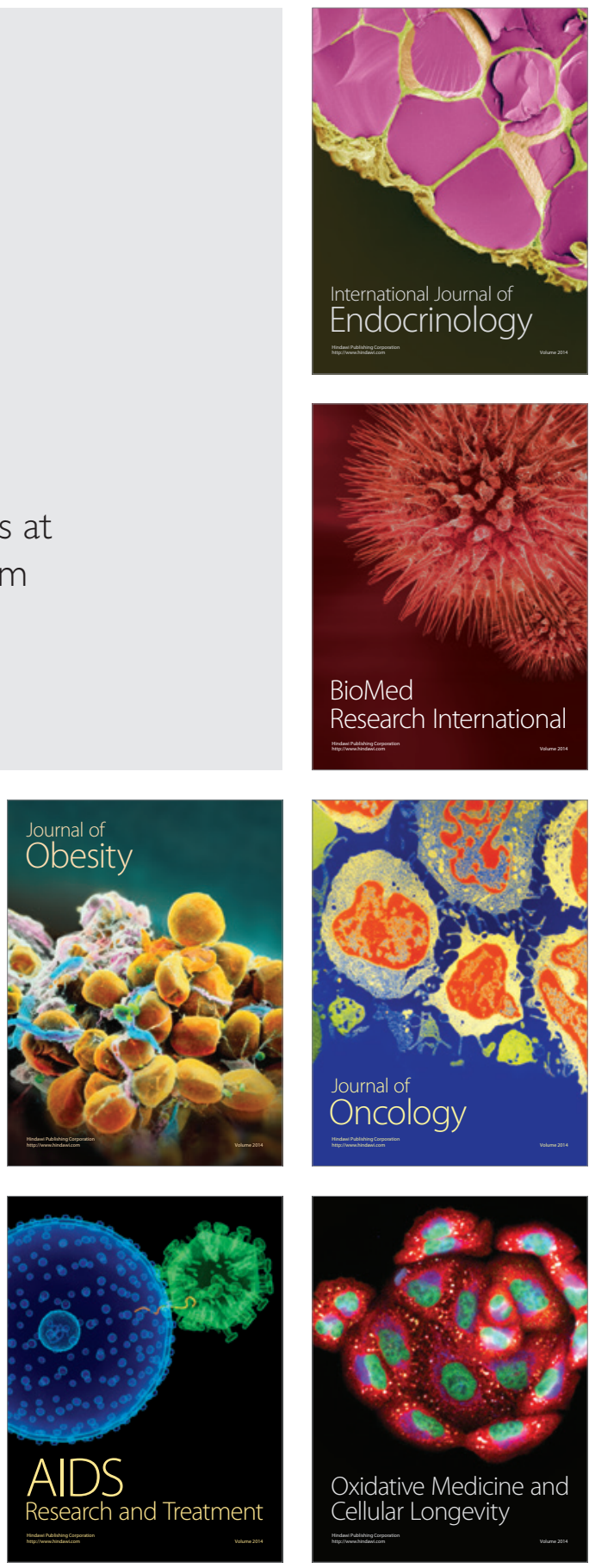